International Mathematical Forum, 1, 2006, no. 12, 593-595

\title{
A universal property of odd degree real Fermat curves
}

\author{
E. Ballico ${ }^{1}$ and R. Ghiloni ${ }^{2}$ \\ Dept. of Mathematics, University of Trento \\ 38050 Povo (TN), Italy \\ ballico@science.unitn.it, ghiloni@science.unitn.it
}

\begin{abstract}
Fix an odd integer $d \geq 1$ and let $F_{d} \subset \mathbb{R}^{2}$ denote the real Fermat curve defined by the equation $x^{d}+y^{d}=1$. Here we prove that $F_{d}$ has the following property: Let $X \subseteq \mathbb{R}^{n}$ be any real algebraic variety. Then there exists a "one-parameter isotopic Nash modification" $\tilde{X}_{t}$ of $X$ such that $\tilde{X}_{0}=X$ and each real algebraic variety $\tilde{X}_{t}, t \neq 0$ may be biregularly embedded into $\left(F_{d}\right)^{n}$.
\end{abstract}

Mathematics Subject Classification: 14P05; 14P20

Keywords: Nash variety; real algebraic variety; Nash map

For the standard definitions of real algebraic varieties, regular maps between two real algebraic varieties and Nash maps, see [1]. For all real algebraic varieties $X, Y$, let $\mathcal{N}(X, Y)$ denote the set of all Nash maps $f: X \rightarrow Y$. Here we just recall the following definitions heavily used in [2] and [3] (see [3], Def. 1.6 and 1.7).

Definition 1. Let $X$ and $\tilde{X}$ be real algebraic varieties and $\phi: \tilde{X} \rightarrow X$ a regular map. We will say that $\phi$ is a weak change of the algebraic structure of $X$ if it is bijective and $\phi^{-1}$ is a Nash map.

Definition 2. Let $X, Z, X^{*}$ be real algebraic varieties, $z_{0} \in Z$ and $\pi: X^{*} \rightarrow Z$ a regular map. A map $\beta: X^{*} \rightarrow X$ is called a weak deformation of $X$ parametrized by $\left(\pi, z_{0}\right)$ if it is regular, $\pi^{-1}\left(z_{0}\right)$ is biregularly isomorphic to $X$, while, for each $z \in Z \backslash\left\{z_{0}\right\}$, the map $\beta \mid \pi^{-1}(z): \pi^{-1}(z) \rightarrow X$ is a weak change of the algebraic structure of $X$.

Hence a regular map $\phi: \tilde{X} \rightarrow X$ between real algebraic varieties is a weak change of the algebraic structure of $X$ if and only if it is a Nash isomorphism. In particular, it must be a homeomorphism for the euclidean topology. Notice that, if $\phi$ is a weak change of the algebraic structure of $X$, then $\phi_{\tilde{X}}^{-1}$ maps nonsingular points to nonsingular points. Hence if $X$ is nonsingular, then $\tilde{X}$ is nonsingular also.

\footnotetext{
${ }^{1}$ The author was partially supported by MIUR and GNSAGA of INdAM (Italy)

${ }^{2}$ The author was partially supported by MIUR and GNSAGA of INdAM (Italy)
} 
Definition 3. Let $M$ be an affine Nash manifold. We say that a Nash map $F$ : $M \times \mathbb{R} \rightarrow M$ is a Nash diffeotopy of $M$ if $F \mid M \times\{t\}$ is a Nash isomorphism for all $t \in \mathbb{R}$ and $F \mid M \times\{0\}: M \times\{0\} \rightarrow M$ is just the isomorphic projection. Let $\mathcal{U}$ be a neighborhood of the identity map $I d_{M}$ in $\mathcal{N}(M, M)$, equipped with the $C^{\infty}$ compact-open topology. We say that $F$ is in $\mathcal{U}$ if $F \mid M \times\{t\} \in \mathcal{U}$ for all $t \in \mathbb{R}$.

Following the proof of [3], Th. 1.8, we are able to obtain the following result which surprised us.

Theorem. For each odd integer $d \geq 1$, the real Fermat curve $F_{d} \subset \mathbb{R}^{2}$ defined by the equation $x^{d}+y^{d}=1$ has the following universal property. Let $X \subseteq \mathbb{R}^{n}$ be any real algebraic variety. Identify $\mathbb{R}^{n} \times \mathbb{R}^{n}$ with $\mathbb{R}^{2 n}$ and $\mathbb{R}^{n}$ with the subspace $\mathbb{R}^{n} \times\{0\}$ of $\mathbb{R}^{2 n}$. Let $\pi: \mathbb{R}^{2 n} \times \mathbb{R} \rightarrow \mathbb{R}$ denote the projection onto the last factor. Choose a neighborhood $\mathcal{U}$ of $I d_{\mathbb{R}^{2 n}}$ in $\mathcal{N}\left(\mathbb{R}^{2 n}, \mathbb{R}^{2 n}\right)$ with respect to the $C^{\infty}$ compactopen topology. Then there exist a real algebraic variety $X^{*} \subseteq \mathbb{R}^{2 n} \times \mathbb{R}$ and a weak deformation $\beta: X^{*} \rightarrow X$ of $X$ parametrized by $\left(\pi \mid X^{*}, 0\right)$ such that:

(a) $X^{*} \cap \pi^{-1}(0)=X$ and $\beta$ extends to a Nash diffeotopy of $\mathbb{R}^{2 n}$ in $\mathcal{U}$;

(b) for each $t \in \mathbb{R} \backslash\{0\}$, the real algebraic variety $\tilde{X}_{t}:=X^{*} \cap \pi^{-1}(t)$ may be biregularly embedded into $\left(F_{d}\right)^{n}$.

Notice that, in the statement of the Theorem, not only we may embed $\tilde{X}_{t}, t \neq 0$, in a product of genus $(d-1)(d-2) / 2$ real algebraic curves all isomorphic to $F_{d}$, but that we may embed it in $\left(F_{d}\right)^{n}$ where the exponent $n$ is the same for all real algebraic varieties which may be embedded in $\mathbb{R}^{n}$. singular ", see [3], Remark 2.3, and use the proof of Theorem given below.

Proof of the Theorem. The proof is just a typographical modification of the proof of [3], Th. 1.8: it is the result that it is surprising, not its proof! Fix an odd integer $d \geq 1$ and $\epsilon>0$. Consider the polynomial $G(x, y, t) \in \mathbb{R}[x, y, t]$ defined by the formula:

$$
G(x, y, t):=\left(\epsilon t x+y\left(1+t^{2}\right)\right)^{d}+\left(-\epsilon t x+y\left(1+t^{2}\right)\right)^{d}-\epsilon^{d} t^{d} .
$$

For each $t_{0} \in \mathbb{R}$, set $D\left[t_{0}\right]:=\left\{(x, y) \in \mathbb{R}^{2} \mid G\left(x, y, t_{0}\right)=0\right\}$. Notice that $D[0]$ is equal to the axis $\{y=0\}$ and, for all $t^{\prime}, t^{\prime \prime} \in \mathbb{R} \backslash\{0\}$, the plane curves $D\left[t^{\prime}\right]$ and $D\left[t^{\prime \prime}\right]$ are biregularly isomorphic: just make a dilatation of the $y$-coordinate. Call $x_{1}, \ldots, x_{n}, y_{1}, \ldots, y_{n}, t$ the coordinates of $\mathbb{R}^{2 n+1}$. Let $G^{*} \subset \mathbb{R}^{2 n+1}$ denote the algebraic subset defined by the $n$ equations $G\left(x_{i}, y_{i}, t\right)=0, i=1, \ldots, n$. Set $X^{*}:=G^{*} \cap\left(X \times \mathbb{R}^{n+1}\right)$. Fix $t \neq 0$. Up to scaling, we may assume that $D[t]$ has $(x+y)^{d}+(-x+y)^{d}=1$ as equation. Notice that the linear isomorphism $\varphi: \mathbb{R}^{2} \rightarrow \mathbb{R}^{2}$ defined by $\varphi(x, y):=(x+y,-x+y)$ induces a biregular isomorphism from $D[t]$ to $F_{d}$. In particular, by the Implicit Function Theorem for Nash maps, there exists a Nash function on $\mathbb{R}$ whose graph coincides with $D[t]$. If $\epsilon$ is sufficiently small, then the proof of [3], Th. 1.8, gives verbatim the proof of the Theorem.

\section{REFERENCES}

[1] J. BOCHNAK - M. COSTE - M.-F. ROY, Real Algebraic Geometry, Springer, Berlin, 1998. MA, 1969. 
[2] R. GHILONI, On the space of real algebraic morphisms, Rend. Mat. Acc. Lincei Ser. IX, (4) (2003), no. 4, 307-317.

[3] R. GHILONI, On the space of morphisms into generic real algebraic varieties, (2005) available at http://www.uni-regensburg.de/Fakultaeten/nat_Fak_I/RAAG/preprints/0098.pdf

Received: October 14, 2005 